\title{
Localization of Autonomous Underwater Vehicles by Floating Acoustic Buoys: A Set-Membership Approach
}

\author{
Andrea Caiti, Senior Member, IEEE, Andrea Garulli, Senior Member, IEEE, Flavio Livide, and
}

Domenico Prattichizzo, Member, IEEE

\begin{abstract}
This paper addresses localization of autonomous underwater vehicles (AUVs) from acoustic time-of-flight measurements received by a field of surface floating buoys. It is assumed that measurements are corrupted by unknown-but-bounded errors, with known bounds. The localization problem is tackled in a set-membership framework and an algorithm is presented, which produces as output the set of admissible AUV positions in a three-dimensional (3-D) space. The algorithm is tailored for a shallow water situation (water depth less than $500 \mathrm{~m}$ ), and accounts for realistic variations of the sound speed profile in sea water. The approach is validated by simulations in which uncertainty models have been obtained from field data at sea. Localization performance of the algorithm are shown comparable with those previously reported in the literature by other approaches who assume knowledge of the statistics of measurement uncertainties. Moreover, guaranteed uncertainty regions associated to nominal position estimates are provided. The proposed algorithms can be used as a viable alternative to more traditional approaches in realistic at-sea conditions.
\end{abstract}

Index Terms-Autonomous robots, localization, set-membership, underwater vehicles.

\section{INTRODUCTION}

$\mathbf{R}$ ECENT years have witnessed an impressive growth in the technology of robotics for undersea exploration. Remotely operated vehicles (ROVs) linked with a tether cable to the mother ship are today a well-established technology routinely used in the off-shore industry. Autonomous underwater vehicles (AUVs) are still more research topics than commercial products; however, they held the promise of being the next significative step in ocean exploration and exploitation, cutting costs and allowing operations that are presently prohibitive from surface ships or by ROVs. One of the problems that prevents commercial applications of AUVs, or at least mitigate their efficiency, is that of vehicle localization: on-board systems, as inertial navigation systems (INS), cannot maintain the requested

\footnotetext{
Manuscript received November 29, 2002; accepted February 3, 2004. This work was supported in part by MURST, Project "Self-localization algorithms for air and maritime vehicles with guaranteed uncertainty bounds." Associate Editor: A. Trucco.

A. Caiti is with the Department of Electrical Systems Automation (DSEA), and the Italian Interuniversity Center of Integrated Systems for the Marine Environment (ISME), University of Pisa, 56100 Pisa, Italy.

A. Garulli and D. Prattichizzo are with the Dipartimento di Ingegneria dell'Informazione, and the Centro per lo Studio dei Sistemi Complessi, Università di Siena, 53100 Siena, Italy.

F. Livide was with the Dipartimento di Ingegneria dell'Informazione, Università di Siena, 53100 Siena, Italy.

Digital Object Identifier 10.1109/JOE.2004.841432
}

accuracy over the desired interval of operation of the system, and are highly expensive.

The general problem of localization of autonomous vehicles has received much attention in the robotic literature, see, e.g., [3], [9], [13]-[15], [18], [24]; however, the peculiarities and constraints of the underwater environment and of usual AUV missions prevent the simple transposition of available techniques for land or aerial vehicles, and require careful study of the implications of each chosen methodology for the underwater system performance [4], [22], [25].

There are several navigation systems currently employed by AUVs researchers. The main nonacoustic approach consists in installing on the AUV a GPS receiver and an INS; the vehicle navigates with the INS, but periodically comes to surface to receive the GPS signal and to recalibrate the INS [26]. The acoustic approaches can be subdivided in the so-called long base-line (LBL) and short or ultra short base-line (SBL) systems. In both cases, the vehicle position is determined on the basis of the acoustic returns detected by a set of receivers. In the LBL case, a set of acoustic transponders is deployed on the seafloor around the perimeter of the area of operation. The transponders are then able to track the vehicle (or the vehicle is able to locate itself with respect to the transponders, depending on where the acoustic emitters are located) with the required accuracy [8]. In SBL systems, a ship follows the AUV at short range with a high-frequency directional emitter able to accurately determine the AUV position with respect to the mother ship; the same system allows for bidirectional communication among the AUV and the ship, so that the AUV navigation system is aware of its current absolute position [23]. All these methods have their merits and drawbacks. Augmented INS requires in any case the setup of sophisticated inertial sensors, and are vehicle-specific (i.e., the same system cannot be employed on more than one vehicle). LBL systems require long time (with associated costs) for deployment and calibration. SBL systems need a ship to follow the vehicle, greatly reducing the cost-effectiveness of an AUV system; in fact, in this case, the vehicle is not really autonomous, but just untethered.

A simpler alternative to LBL systems has been recently investigated, consisting in installing acoustic receivers/emitters on surface freely floating buoys [2]; on the buoys GPS receivers with radio interconnection are also installed. The vehicle is located through simple time-of-flight measurements of acoustic pings from each buoy, with one of the buoys (or an additional remote station) acting as "master," i.e., collecting information 


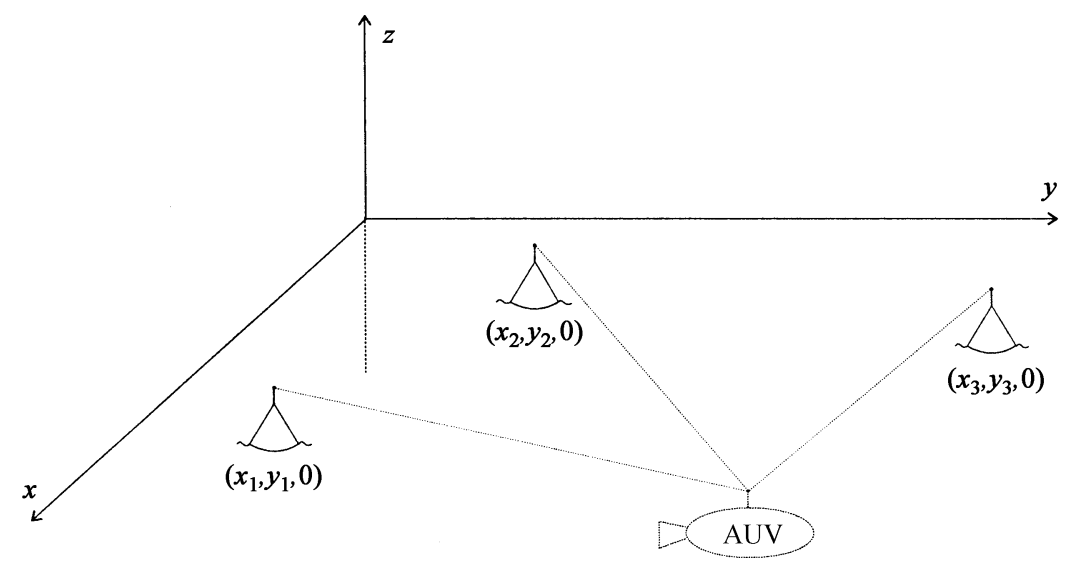

Fig. 1. Framework for AUV localization.

from the other buoys and determining the absolute position of the vehicle. Alternatively, each buoy emits at regular time intervals a ping with the coded information of its GPS position. The vehicle listens for the pings, and again it determines its absolute position from time-of-flight measurements. In this second alternative, the floating buoys system has the ambition of becoming a true underwater GPS system, affordable, easy to deploy and recover, autonomous during its time of operation. The localization and tracking performance of such a system has been recently investigated by several authors [2], [8], [10], [20], [21]; in all these cases, the algorithms have considered measurements affected by Gaussian-distributed noise, and have determined the resulting localization uncertainties through Monte Carlo analysis or nonlinear transformation techniques [12].

In this paper, a different approach is proposed and investigated, based on the assumption that measurement errors are unknown but bounded, with known bounds. Tools from set-membership estimation theory [16], [17] are employed to determine the admissible region in space in which the vehicle is located. Though this approach can be easily generalized to the deep water case, our discussion and implementation refers only to the shallow water case (water depth less than $500 \mathrm{~m}$ ), which is the most interesting from the point of view of AUV applications, and the most challenging due to the presence of multipath effects. The performance of the algorithm has been tested in simulation, considering buoys dislocation similar to those employed in [8] and [21], to allow comparison of results. The error measurement characteristics have been taken from field experiments at sea [19]-[21], in order to obtain a realistic characterization of the proposed approach. The results obtained show that the set-membership approach has at least similar performance to that obtained by standard triangulation methods. It is remarked, however, that the main advantage of the set-membership approach is the determination of regions where the vehicle is guaranteed to belong, with the sole prior knowledge of the worst-case bound on the measurement error. Partial results on this localization approach have been presented in [6]. In this paper, the localization algorithm is described in detail also in the varying sound speed case, and comparison is made with results obtained by a standard triangulation approach. The use of set-membership information in AUV tracking and filtering problems has been proposed and outlined in [5] and [7].

The paper is organized as follows: in Section II, the problem is formally stated, some relevant properties of acoustic propagation in the ocean are briefly recalled and the methodological set-membership approach is presented; in Section III the proposed set-membership localization algorithm is described in detail; in Section IV a pseudoMonte Carlo analysis of performance of the algorithm is presented for the cases of winter and summer sound speed profile conditions; finally some conclusions are given in Section V.

\section{Problem Statement and General METHODOLOGICAL APPROACH}

Let us consider the case in which $n$ buoys are placed in arbitrary positions on the sea surface over an area of interest. An absolute earth reference system $(x, y, z)$ is assumed, with $z=0$ on the water surface, and the $z$-axis pointing upward from the sea surface (see Fig. 1). Each buoy position $\left(x_{i}, y_{i}, 0\right)$ is assumed to be known. In practice any buoy position will be known at (differential) GPS accuracy; the uncertainty in the buoy position can be treated as an additional uncertainty in the buoy measurement. The buoys are allowed to move freely; however, since their movement will be due to waves and current, with a dynamic much slower with respect to the time scale of the travelling acoustic signals, it is assumed that the buoys nominal position is fixed, and the buoys possible movement is treated again as additional uncertainty in the measurement. Without loss of generality, it is assumed that the vehicle transmits, at known synchronized times, an acoustic ping, which is received at the buoys. The symmetric situations, in which the buoys transmit pings received by the AUV, or the case of monostatic transmission/reception (i.e., when each buoy transmits a ping and receives the echo of the ping from a transponder installed on the AUV), can be treated in a similar way. The (non trivial) low-level signal processing needed for each buoy to detect the signal transmitted by the AUV is beyond the scope of the present paper. Note, however, that fake measurements due to false detections can be viewed as outliers and treated accordingly (a possible method for detecting outliers in the set-membership set- 
ting is given in [1]). So, it is assumed that the received signals are suitably processed so that, after reception of each echo, the measurement $s_{i}$ of the one-way travel time of the emitted ping from the AUV is available to the $i$ th buoy. Each measurement is affected by an unknown-but-bounded error $e_{i}$, which accounts for all the uncertainties (due to synchronization errors, noisy signals, D-GPS accuracy, buoy/vehicle motion between transmission and reception of the same ping, etc.), i.e.

$$
s_{i}=\tilde{s}_{i}+e_{i}, \quad\left|e_{i}\right| \leq E_{i}, \quad i=1, \ldots, n
$$

being $\tilde{s}_{i}$ the measurement when no uncertainties are present, and $E_{i}$ a known bound on the measurement uncertainty. Note that the known bound may be allowed to change with time, for instance in presence of time-base drifts. The proposed methodology can handle this situation without any modification, except for adapting in time the bound $E_{i}$. In this paper the static case is treated to simplify the presentation, and it is assumed that all the possible uncertainties have been appropriately bounded at each time.

The sound speed $c(x, y, z)$ in the area of interest is assumed known. Though the proposed approach can deal with the general case, in the following the most common situation in which the sound speed varies only with depth in the area of interest is considered, i.e., $c(x, y, z)=c(z)$. Acoustic propagation is modeled with ray path theory [11], including multipath effects, and considering lossless reflection at the water surface and seafloor boundaries. Note that, for the purpose of the localization algorithm, only the acoustic ray paths, and not the intensities of the acoustic signals, need to be computed, so the assumptions made are not restrictive. The sources/receivers are considered omnidirectional, i.e., they emit/receive acoustic rays in every direction below the plane $(x, y)$ (the sea surface). Propagation along the sea surface boundary has not been considered, since in practice these rays are very much attenuated and scattered by wave motion and bubbles. Each ray arriving at the $i$ th buoy with angle $\bar{\theta}\left(x_{i}, y_{i}, 0\right)$ with respect to the plane $(x, y)$ has travelled along a path respecting Snell's law at every point $(x, y, z)$, i.e.

$$
\frac{\cos (\theta(x, y, z))}{c(z)}=\frac{\cos \left(\bar{\theta}\left(x_{i}, y_{i}, 0\right)\right)}{c(0)}
$$

where $\theta(x, y, z)$ is the angle between the ray and the $(x, y)$ plane, at the generic point $(x, y, z)$. The measurement of the $i$ th buoy, once converted from time to distance taking into account the sound speed profile $c(z)$, and considering the bounded uncertainty (1), defines a region $S_{i}$ of the admissible space $B_{0}$ in which the vehicle is located. The space $B_{0}$ is bounded by the sea surface and sea bottom, and by the defined extension of the area of interest; in the description of the algorithm it will be assumed that the bottom is flat, however variable bathymetry can be easily considered. The buoys are located inside $B_{0}$. Merging the information from all the available buoys, the region $V$ in space in which the vehicle is positioned is given by

$$
V=B_{0} \bigcap\left(\bigcap_{i=1}^{n} S_{i}\right) .
$$

This is usually addressed as feasible position set in the setmembership literature. The region $V$ may have a complicated geometrical shape, making its exact computation infeasible. The objective of the set-membership localization algorithm described in the next section is to determine a guaranteed approximation of $V$, i.e., a set $R$ such that $R \supseteq V$. If $\mathbf{p}=$ $\left(x_{p}, y_{p}, z_{p}\right)^{\prime}$ is the true position of the AUV to be estimated, this guarantees that $\mathbf{p} \in R$.

In order to obtain tractable algorithms, it is customary to select a class of regions $R$ of simple structure. In this paper, boxes will be considered as approximating regions. A box is defined as

$$
B=\{(x, y, z): \underline{x} \leq x \leq \bar{x}, \quad \leq \bar{y}, \quad \underline{z} \leq z \leq \bar{z}\} .
$$

Boxes are convenient geometrical objects to use, since efficient set approximation procedures can be employed for their computation. Clearly, one is interested in obtaining the box of smallest volume containing the set $V$. Again, this can be a very difficult task, due to the involved structure of the regions generated by the intersection of the sets $S_{i}$. Hence, suboptimal solutions are usually sought. The one chosen in this paper is discussed in the next section.

\section{The LOCALIZATION ALGORITHM}

\section{A. Constant Sound Speed Profile}

In order to give a clear understanding of the set-membership localization algorithm, it will be firstly described for the case of sound speed constant with depth, i.e., $c(z)=c$. Note that this simplified condition does frequently occur in the shallow ocean, particularly in winter periods when storms and the lack of prolonged solar radiation make the sea temperature homogeneous over the water column. Thus, this situation is often referred to as "winter conditions" [11]. In the presence of winter conditions, the acoustic rays propagate along straight paths, so that the distance of the vehicle from the $i$ th buoy can be simply computed from the time-of-flight measurement (1) as

$d_{i}=\frac{s_{i}}{c}=\frac{\tilde{s}_{i}+e_{i}}{c}=\tilde{d}_{i}+\varepsilon_{i},\left|\varepsilon_{i}\right| \leq \frac{E_{i}}{c}=F_{i}, i=1, \ldots, n$.

The set of vehicle positions compatible with the measurement $d_{i}$ is a spherical cap $L_{i}$ of center $\mathbf{c}_{i}=\left(x_{i}, y_{i}, 0\right)$ (the buoy position) and of internal and external radii $R_{1 i}=d_{i}-F_{i}$ and $R_{2 i}=d_{i}+F_{i}$, respectively, i.e.

$$
\begin{aligned}
L_{i}=\left\{(x, y, z):\left(d_{i}-F_{i}\right)^{2}\right. & \leq\left(x-x_{i}\right)^{2} \\
& \left.+\left(y-y_{i}\right)^{2}+z^{2} \leq\left(d_{i}+F_{i}\right)^{2}\right\} .
\end{aligned}
$$

The set $V$ of vehicle positions compatible with the measurements from all the buoys is obtained by substituting the terms $S_{i}$ with $L_{i}$ in (3).

To find a box $B$ containing the set $V$, the following procedure is employed. At first, the intersections of $L_{i}$ and $L_{j}$ for all the possible couples $i, j, i \neq j$ are considered (see Fig. 2). For each intersection, the aim is to compute the minimum volume box containing $L_{i} \cap L_{j}$, in a new reference system.

Let $\mathbf{p}$ be a point in the earth absolute reference system. Consider the new reference frame $(\tilde{x}, \tilde{y}, \tilde{z})$, defined by the change 


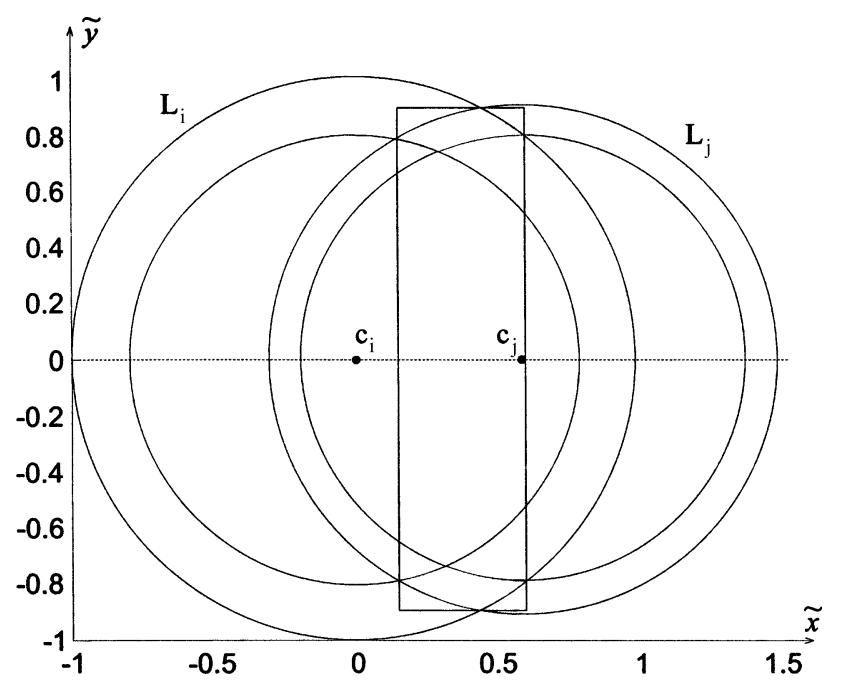

Fig. 2. Intersection between two spherical caps $L_{i}, L_{j}$ (planar view); the admissible regions for the vehicles are the shaded regions. The rectangle represents the box of minimal volume containing the admissible region. The new coordinate system has the $\tilde{x}$-axis oriented along the line through the buoys $i, j$ (the centers of the spherical caps).

of coordinates $\mathbf{p}=A \tilde{\mathbf{p}}+\mathbf{c}_{i}$, where $\tilde{\mathbf{p}}$ is the generic vector in the new coordinates. The first column of the matrix $A$ is given by the unitary vector $\mathbf{p}_{c}=\mathbf{c}_{i}-\mathbf{c}_{j} /\left\|\mathbf{c}_{i}-\mathbf{c}_{j}\right\|$ and the other two columns can be taken as any vectors orthonormal to $\mathbf{p}_{c}$; in particular, one can choose the $\tilde{z}$ axis equal to that of the absolute reference system (an admissible choice, since $\mathbf{p}_{c}$ lies on the sea surface plane). The new coordinate system has the $\tilde{x}$ axis aligned with the vector $\mathbf{p}_{c}$ joining the centers of the two spherical caps, and origin in one of the two centers. Since the intersection problem has cylindrical symmetry with respect to the $\mathbf{p}_{c}$-oriented axis, the two caps can be projected on the plane generated by $\mathbf{p}_{c}$ and by one of the other two axis. The projection on this plane gives origin to two circular anuli $H_{i}$ and $H_{j}$. The rectangle with minimal area containing the intersection of $H_{i}$ and $H_{j}$ is identified by determining the following quantities

$$
\begin{aligned}
\tilde{x}_{\text {min }} & =\min _{\tilde{\mathbf{p}} \in H_{i} \cap H_{j}} \tilde{x} \\
\tilde{x}_{\text {max }} & =\max _{\tilde{\mathbf{p}} \in H_{i} \cap H_{j}} \tilde{x} \\
r & =\max _{\tilde{\mathbf{p}} \in H_{i} \cap H_{j}} \tilde{y} .
\end{aligned}
$$

This can be done by simple enumeration of the intersection points among the two anuli, and of each anulus with the $\tilde{x}$ axis. The rectangle thus obtained (see Fig. 2) can then be rotated around the vector $\mathbf{p}_{c}$ to generate a cylinder which includes the admissible region, of radius $r$ and of height $h=x_{\max }-x_{\min }$. This cylinder can in turn be bounded by a box $\tilde{B}$ defined as in (4), with

$$
\begin{array}{ll}
\underline{x}=x_{\min } & \bar{x}=x_{\max } \\
\underline{y}=\underline{z}=-r & \bar{y}=\bar{z}=r .
\end{array}
$$

At this stage, the coordinate transformation $\mathbf{p}=A \tilde{\mathbf{p}}+\mathbf{c}_{i}$ can be inverted, and the box described by (4) and (7) can be written in the earth absolute reference frame as a set of six linear inequalities in the form

$$
M \mathbf{p} \leq \mathbf{b}, \quad M \in \mathbb{R}^{6 \times 3} .
$$

Let $B_{k}, k=1, \ldots, n(n-1) / 2$, be the set of all boxes determined by considering all the possible pairs $L_{i}, L_{j}, i \neq j$. Hence, each box defines a set of constraints as in (8) in the earth reference system, that are now written as

$$
M_{k} \mathbf{p}=\mathbf{b}_{k}, \quad k=1, \ldots, \frac{n(n-1)}{2} .
$$

Recall that $B_{0}$ is the whole region of space where the AUV can be positioned. Therefore, a box $B$ containing the feasible set $V$ is given by the box of minimal volume including the intersection of the boxes $B_{k}, k=0, \ldots, n(n-1) / 2$. In the earth reference frame, the box containing such intersection is simply found by solving the following six linear programming problems

$$
\begin{aligned}
& \min (x), \max (x), \min (y), \max (y), \min (z), \max (z) \\
& \text { subject to constraints } \\
& M_{k} \mathbf{p}=\mathbf{b}_{k}, \quad k=0, \ldots, \frac{n(n-1)}{2} .
\end{aligned}
$$

It is worth observing that the box provided by (9) is not necessarily the minimum volume box containing $V$, since it is not true that a box of minimal volume in a given coordinate system has minimal volume for any coordinate system. The size of the approximating box can be reduced by considering different reference systems and repeating iteratively the above approximation algorithm. The following recursive procedure has been implemented.

a) solve (9) in the absolute coordinate system obtaining a box $\hat{B}$; set $B \leftarrow \hat{B}$;

b) for every buoy $i, i=1, \ldots, n$, do:

- compute a coordinate transformation setting the $x$ axis along the direction joining the center of $B$ with the buoy position;

- solve (9) in the new coordinate system obtaining a box $\hat{B}$; if volume $(\hat{B})<\operatorname{volume}(B)$ set $B \leftarrow \hat{B}$

c) if volume $(B)$ has not changed during the last step, exit with the result $B$; otherwise, go to step $b$ ).

Clearly this iterated procedure is suboptimal, since it does not check the condition of minimal volume along all possible coordinate transformations. However, it does check the minimality conditions over a set of privileged directions, that are those joining the buoy positions with the best estimate currently available of the AUV position. Note that the whole procedure is computationally feasible as long as the number of buoys is not too large. In practical systems, the number $n$ of buoys is often limited to 3 or 4; as long as the number of linear constraints is of the order of 500 (i.e., the number of buoys is kept below 15) standard available programs can efficiently perform the computation.

\section{B. Variable Sound Speed Profile}

The more general case of sound speed profile $c(z)$ varying with depth is treated with the same geometrical machinery; the 


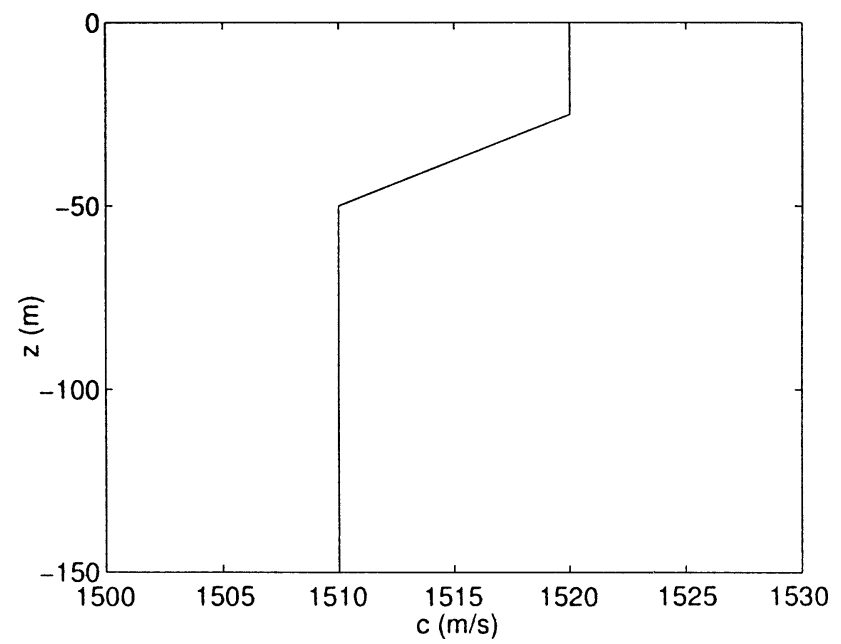

Fig. 3. Sound speed profile with depth in summer conditions, water depth $150 \mathrm{~m}$.

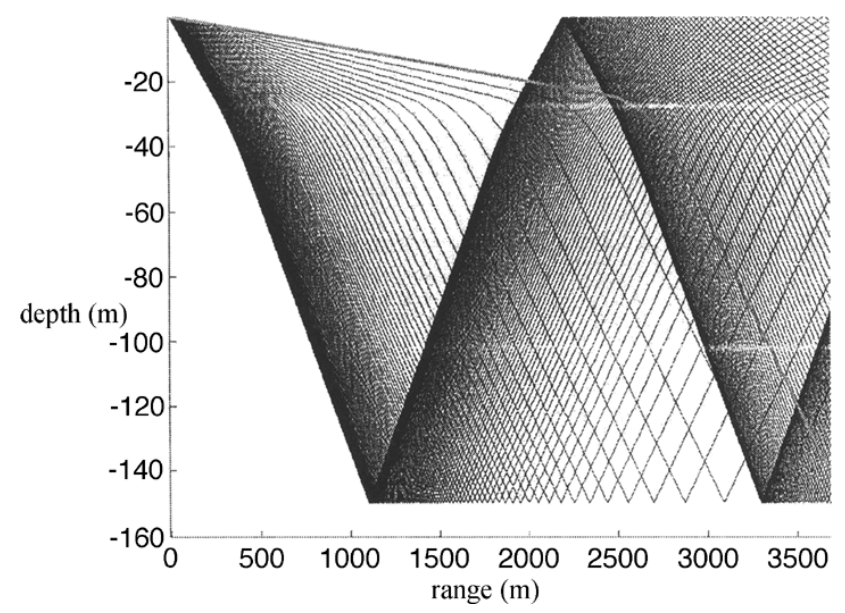

Fig. 4. Ray paths from the emission point at the sea surface, corresponding to the sound speed profile of Fig. 3; both axis are in meters, water depth is $150 \mathrm{~m}$.

main difference is due to the determination of the spherical cap $L_{i}$ of admissible AUV positions, obtained from the $i$ th buoy on the basis of the time measurement $s_{i}(2)$. In fact, the admissible region corresponding to each measurement will be determined as the intersection of a set of spherical caps. In order to illustrate the process, let us consider a typical "summer profile" of sound speed (Fig. 3), in which a warm layer (higher sound speed) is present, due to the effect of solar radiation. In Fig. 4 the acoustic propagation ray paths corresponding to the summer profile are shown (since the propagation has cylindrical symmetry around the $z$ axis, only a planar figure is presented). The upper ray in the figure (thicker line) is the limiting ray, i.e., the ray characterized by the smallest angle $\theta$ with the sea surface at the receiver location (0.01 rad in this case). It can be seen that, due to Snell's law, the rays are bending toward the regions of minima in the sound speed profile, and the propagation occurs through bounces on the seafloor and on the sea surface. The points compatible with the measurement are those reached after a time $s_{i}$ along some ray, and such that there does not exist any other ray path that can reach the same point in a shorter time. These sets of points form isotemporal curves that can be divided in five classes (which do

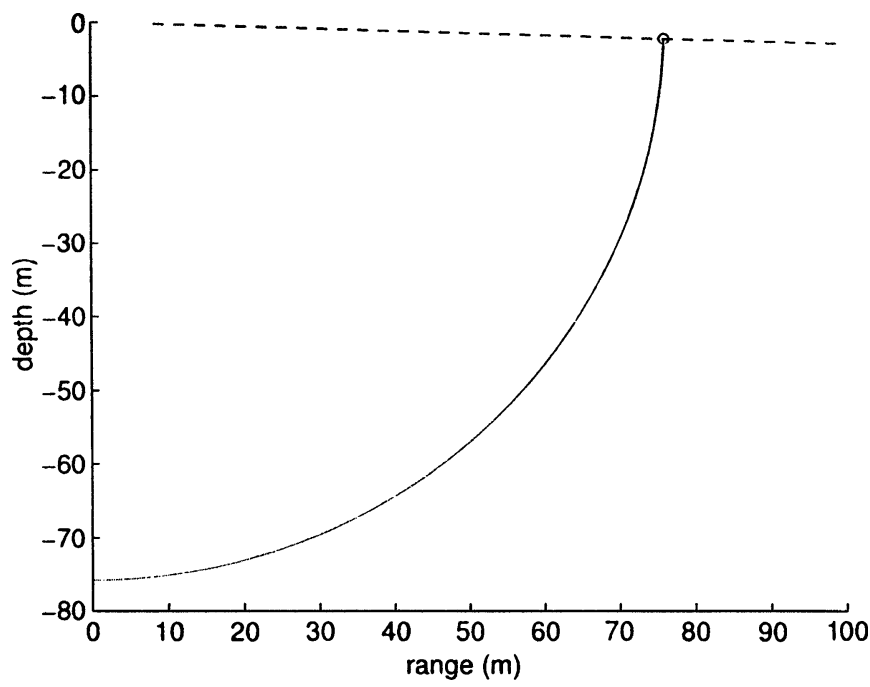

Fig. 5. An isotemporal curve, i.e., a set of points that are reached by the acoustic rays coming from the buoy in position $(0,0)$ at the same time, and before any other acoustic ray has reached them. The figure illustrates class 1 , in which all the points in the isotemporal curve are reached by rays that do not reflect on the sea surface or the sea bottom. The curve has been obtained for the summer sound speed profile of Fig. 3.

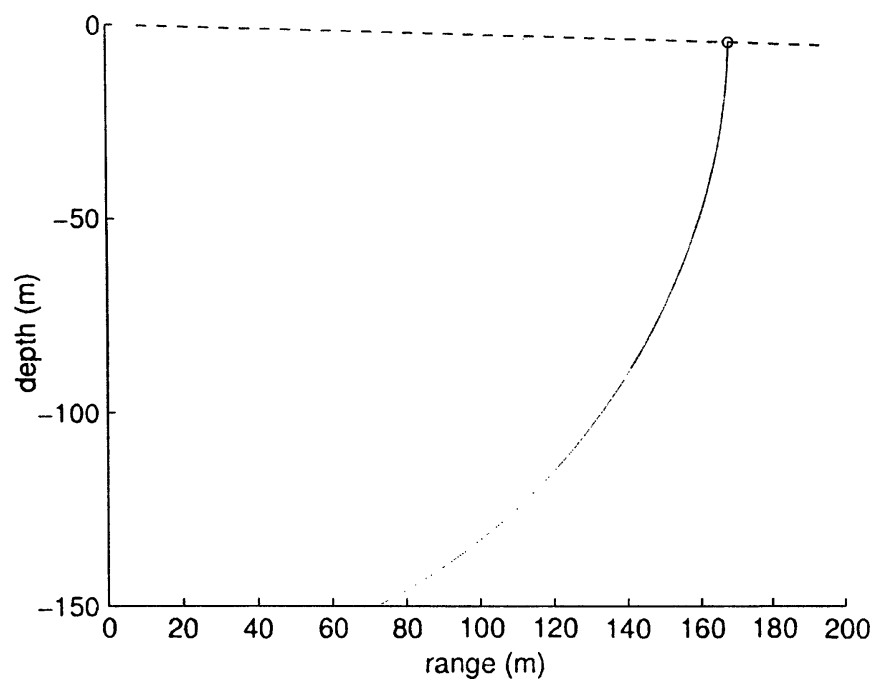

Fig. 6. Class 2 isotemporal curve. The curve is limited by the bottom and by the limiting ray (dashed).

not depend on the specific sound speed profile). These classes are illustrated in Figs. 5-9 (the dashed curve always represents the limiting ray).

For each of the five classes of curves, when the worst-case uncertainty is taken into account, specific admissible regions $S_{i}$ are generated. Note that these regions are not anymore spherical caps; moreover, in some cases the regions are discontinuous. In order to apply the algorithm described in the constant velocity case, the admissible regions $S_{i}$ are bounded by spherical caps, all having centers on the $z$ axis at the $\left(x_{i}, y_{i}\right)$ coordinates of the $i$ th buoy. An example of the construction of such spherical caps is illustrated in Figs. 10, 11, for class 3 depicted in Fig. 7. Fig. 10 shows how the region $S_{i}$ (shaded) is contained in the intersection of three spherical caps (the thicker lines). The leftmost sphere (the one passing through $\mathrm{A}$ ) is the one with maximum 


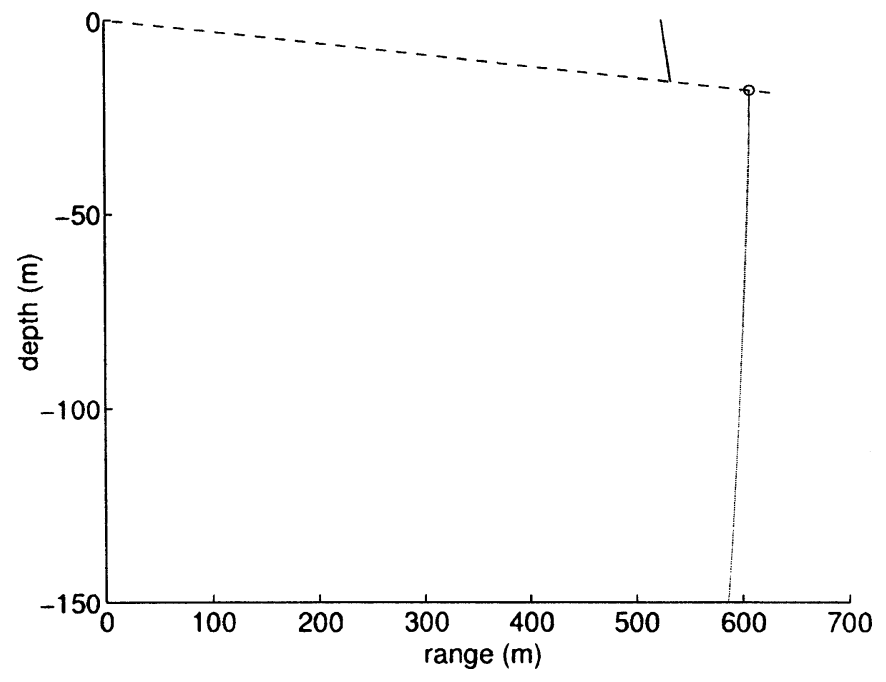

Fig. 7. Class 3 isotemporal curve. The part of the curve above the limiting ray is due to rays that are surface reflected.

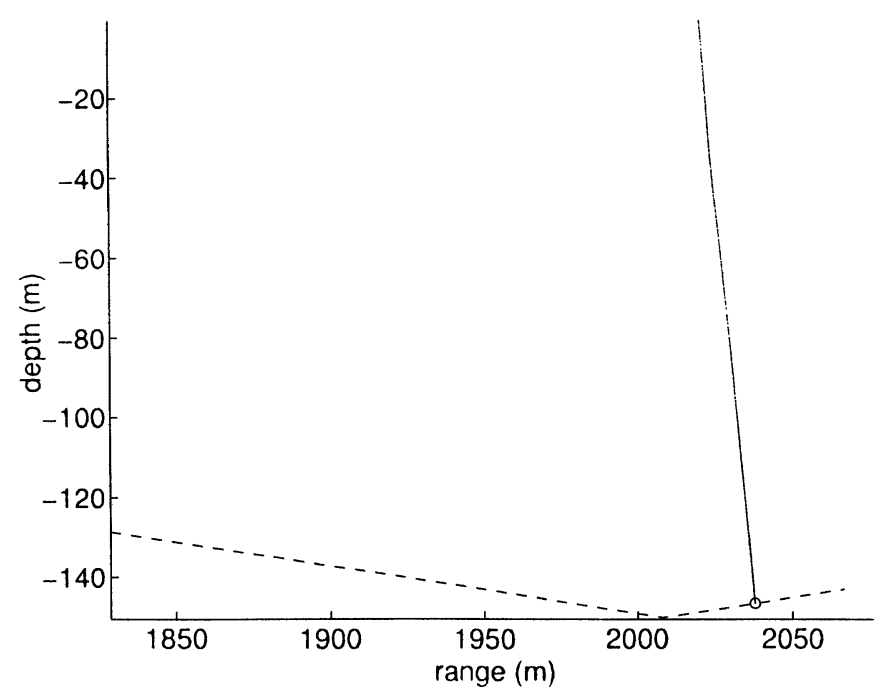

Fig. 8. Class 4 isotemporal curve: the curve is limited by the reflection of the limiting ray on the sea bottom.

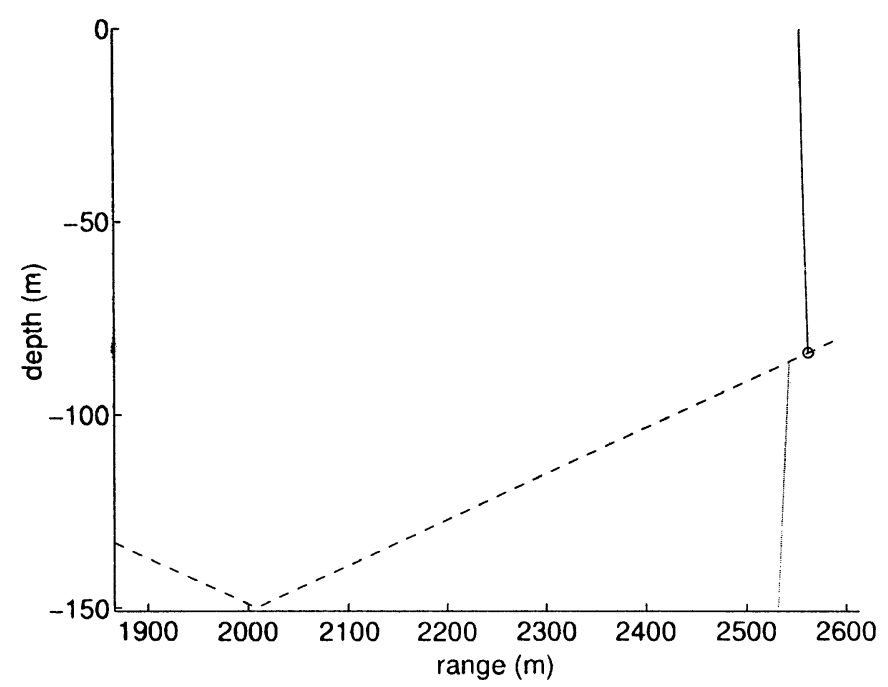

Fig. 9. Class 5 isotemporal curve: the part of curve below the limiting ray is due to bottom reflected rays.

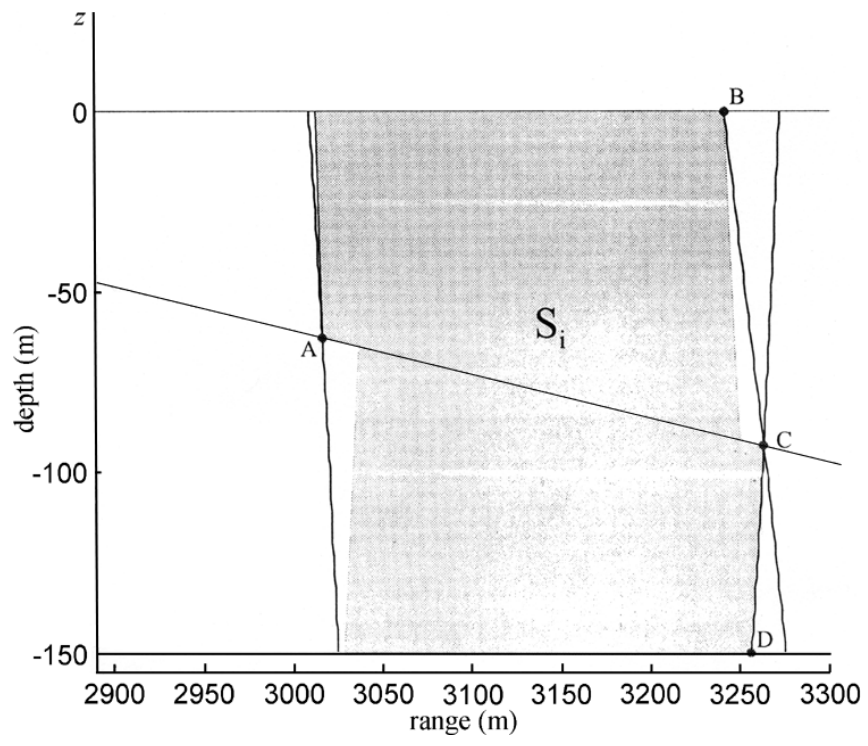

Fig. 10. Admissible region for the class of Fig. 7. The shaded area is the admissible region $S_{i}$ compatible with the $i$ th measurement. The three circumferences passing, respectively, through the points A, B and C, C and D, bound the admissible region. The figure has cylindrical symmetry around the $z$ axis, so the circumferences are spheres in the 3-D case.

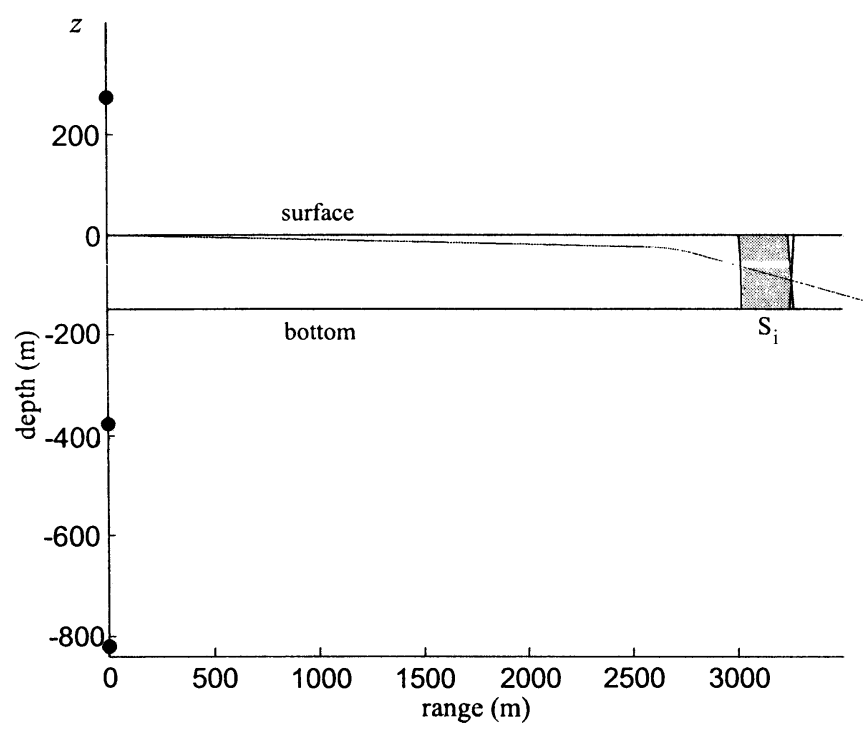

Fig. 11. A broader view of Fig. 10: the dots on the $z$ axis are the centers of the three circumferences bounding $S_{i}$, acting as virtual buoys. The true buoy is located in the origin of the reference system.

radius and such that $S_{i}$ lies all outside the sphere. The other two spheres are generated by rotation around the $z$ axis of the circumferences passing through points $\mathrm{B}$ and $\mathrm{C}$, and $\mathrm{C}$ and $\mathrm{D}$, respectively. For each buoy $i$ a set of spherical caps $L_{i k}$, $k=1, \ldots, \bar{k}_{i}, \bar{k}_{i} \leq 3$ is generated, each with center in a point $\left(x_{i}, y_{i}, z_{i k}\right)$, and such that the intersection of these spherical caps contains the admissible region $S_{i}$. This means that the points $\left(x_{i}, y_{i}, z_{i k}\right)$ can be treated as the positions of virtual buoys, whose measurements generate as admissible region a spherical cap. Notice that the virtual buoys may be located below the sea bottom or above the sea surface (see Fig. 11). 
As a consequence of the above reasoning, one has that the feasible position set $V$ is contained in the intersection of all the feasible caps generated to approximate the sets $S_{i}$, i.e.

$$
V \subset \bigcap_{i=1}^{n} \bigcap_{k=1}^{\bar{k}_{i}} L_{i k}
$$

This is a situation similar to the one treated in the "winter conditions" case discussed above, so the same algorithm can be applied to the set of all virtual buoys in order to generate the bounding box $B$ containing the region compatible with the measurements of the $n$ real buoys. Note that one has to process at most $3 n$ measurements from virtual buoys, thus leading once again to computationally tractable linear programming problems.

It is important to remark that in the general arbitrary sound speed profile case the algorithm proposed has an additional suboptimal step with respect to the constant sound speed profile case: the procedure for building spherical caps limiting the feasible set of vehicle positions; this procedure is due to the variable geometrical shape of the feasible set, that, as illustrated, may even be discontinuous. This additional step (not needed in the constant profile case, where the feasible regions $S_{i}$ are already spherical caps) introduces a further degree of conservativeness in the localization bounds obtained with a variable sound speed profile, with respect to those obtained with a constant sound speed profile.

\section{LOCALIZATION AlgorithM: PERFORMANCE ANALYSIS}

Performance analysis of the set-membership localization algorithm through a pseudoMonte Carlo simulation approach is now reported. This simulative exercise has several objectives. The first is to analyze if the set-membership approach leads to localization uncertainties that exhibit qualitatively the same spatial distribution patterns of standard triangulation algorithms, and quantitatively the same order of magnitude (or if there are significant changes with respect to the standard approach, for the better or for the worse); to this aim, the same geometrical configuration and system and uncertainty parameters reported in [21] have been used; note that other systems may have different characteristics: in particular, the system described in [2], operating at higher frequencies and at smaller ranges, has uncertainty of approximately one order of magnitude less than that of [21]. The second objective is to analyze the change in the algorithm performance when different probability distributions are used in the synthetic data generation: it is generally claimed that the set-membership approach is better suited to the cases in which the measurement uncertainties have uniform probability distribution, and since the true error probability distribution is not known, it is important to check through simulation if there is any significant degradation of performance when different probability distributions are considered. The Gaussian distribution, as the most widely used in the modeling of uncertainties, has been taken as term of comparison. The third objective of the simulations is the quantitative analysis of the differences in algorithm performance between the "winter" and the "summer" condition case, since in the latter case the algorithm provides more conservative bounds on the feasible regions. The implemented algorithm has the potential of analyzing the localization error as a function of the number of buoys and of the buoys geometry; however, optimization with respect to these system and geometrical parameters (in dependence of different environmental conditions and localization precision requirements) is a complex and challenging problem which deserves deeper investigations, and it is not treated here. The chosen geometrical configuration has been selected uniquely to allow comparison with the results in [21].

The rules of the game in the simulations have been set as follows. An admissible region of $30 \times 30 \mathrm{Km}$ in the $(x, y)$ plane is considered, with water depth of $150 \mathrm{~m}$. Three buoys are considered, placed as vertex of an equilateral triangle of $16 \mathrm{Km}$ side. The admissible region has been gridded in the $(x, y)$ plane at $1 \mathrm{Km}$ spacing. The depth of the vehicle has been kept at $75 \mathrm{~m}$ at any point of the grid. For each point of the grid, 10 different measurement realizations have been generated, and the localization algorithm applied. The nominal localization error and the worst-case localization error, averaged over the 10 realizations, are reported for each point. The nominal error is computed as the distance between the center of the bounding box $B$ and the true vehicle position. The worst-case localization error is computed as the semidiagonal of the bounding box $B$. Note that, due to symmetry of the buoy configuration, one of the buoy is taken at the origin of the coordinate system, and another one on the $x$ axis. The uncertainty regions for $x<0$, or $y<0$ (or both) can be obtained in this case by trivial symmetry operations.

In the winter condition case, the sound speed has been taken as $1500 \mathrm{~m} / \mathrm{s}$ everywhere. The measurement realizations have been obtained first assuming a uniformly distributed measurement noise $\varepsilon_{i}$ [see (5)] within the interval [-100 100] m, with independent identical distribution (i.i.d.) for each buoy; in a second case, a Gaussian distributed measurement noise has been assumed (again, i.i.d. for each buoy), with zero mean and standard deviation $\sigma=33 \mathrm{~m}$. In both cases, the algorithm assumes the knowledge of the noise bound $F_{i}=100 \mathrm{~m}$ (roughly equal to $3 \sigma$ in the Gaussian case). Note that a $33 \mathrm{~m}$ standard deviation, with a constant sound speed of $1500 \mathrm{~m} / \mathrm{s}$, corresponds to a standard deviation of $22 \mathrm{~ms}$ in the time of arrival measurement error. Mozzone et al. [21] have reported experimental data with a similar geometrical configuration and transmission in the 1.9-3.5 $\mathrm{kHz}$ range. They estimated a two-way time of flight variance $\sigma$ between 30 and $60 \mathrm{~ms}$ (depending on the buoys). The noise bound used in our simulations has been chosen by picking an average value of $\sigma=45 \mathrm{~ms}$, according to Mozzone et al. [21], and then taking approximately half of that value to account for the one-way time of flight configuration in our setting.

Fig. 12 and 13 show the nominal and worst-case localization errors, respectively, obtained assuming uniformly distributed measurement noise. Fig. 14 and 15 report the corresponding results obtained with Gaussian distributed measurement noise. The results of the two cases are first compared one against the other. The nominal error in the case of uniformly distributed uncertainties is larger than the one obtained with Gaussian distributed uncertainties; however the worst-case errors are smaller in the uniform distribution case. There is an intuitive 


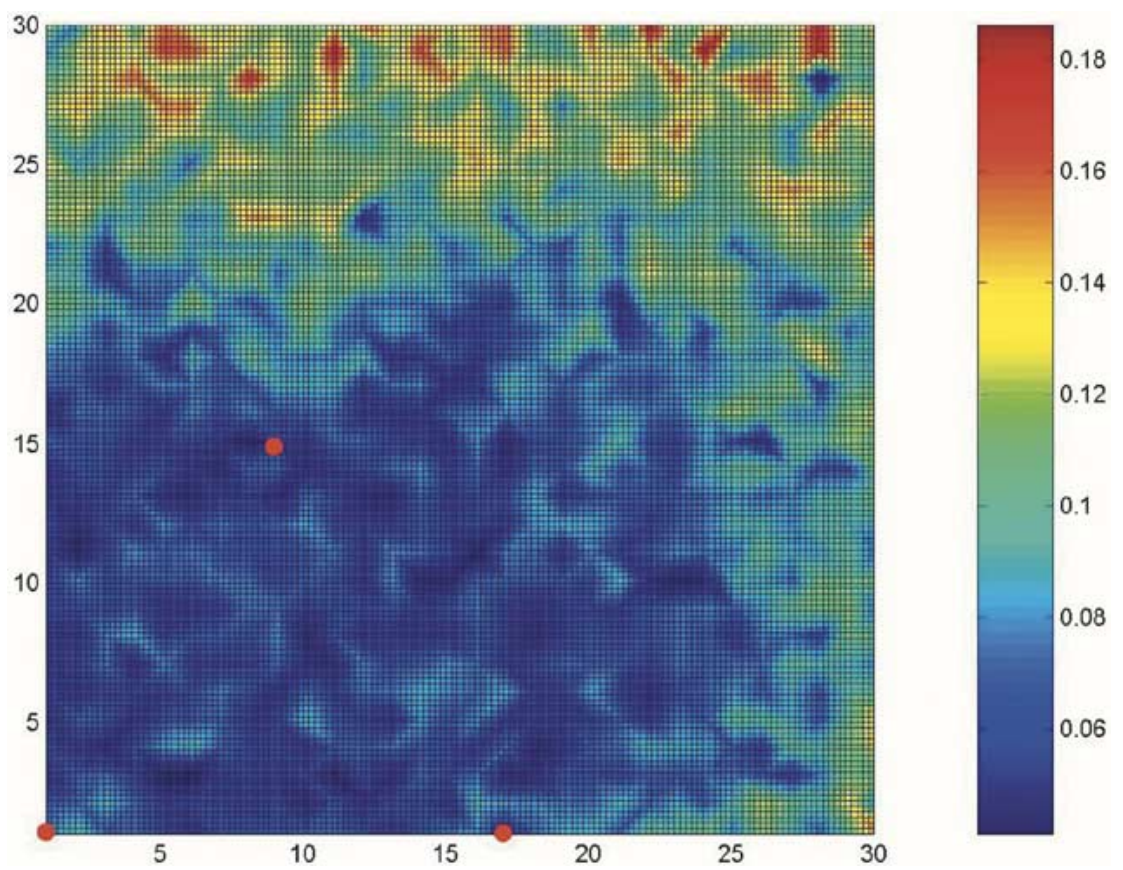

Fig. 12. Color-coded average nominal error over the admissible region as a function of $(x, y)$ position in the case of uniformly distributed measurement error, winter conditions. The error chromatic scale is in $\mathrm{Km}$. Buoy locations are the red circles. Scales on the $x$ and $y$ axis are in $\mathrm{Km}$.
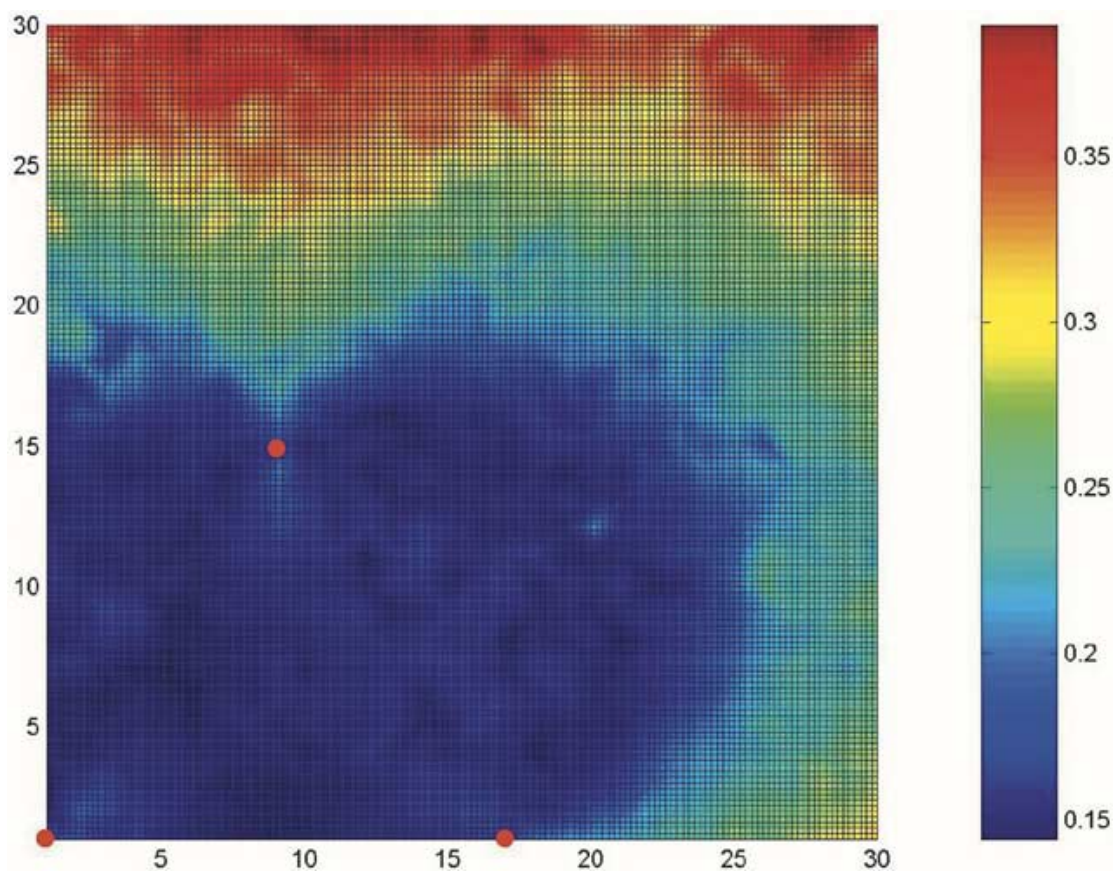

Fig. 13. Color-coded average worst-case error over the admissible region as a function of $(x, y)$ position in the case of uniformly distributed measurement error, winter conditions. The error chromatic scale is in $\mathrm{Km}$. Buoy locations are the red circles. Scales on the $x$ and $y$ axis are in $\mathrm{Km}$.

explanation for this result. When the error is Gaussian distributed, the dispersion of the samples from the mean value is less than in the case of uniform distribution. This implies that the samples in the Gaussian case will be more concentrated around the true value, and the associated uncertainty regions will greatly overlap; so the center of the bounding box $B$ computed by the set-membership algorithm will be relatively close to the true value, but the worst-case uncertainty will not be significantly reduced by the intersection operation, because of the overlap of the uncertainty regions. In the uniform distri- bution case, the samples are more scattered, causing the center of the bounding box to be more distant from the true position, but allowing a greater reduction of the worst case uncertainty through the intersection operation. Note also that, for the same reason underlined above, the nominal error pattern is in both cases much more irregular than the worst-case error pattern. It is likely that a consistently larger number of realizations is needed to smooth out also the nominal error pattern.

The qualitative aspect of the (worst-case) error pattern distribution is equivalent in both cases, showing that accuracy de- 


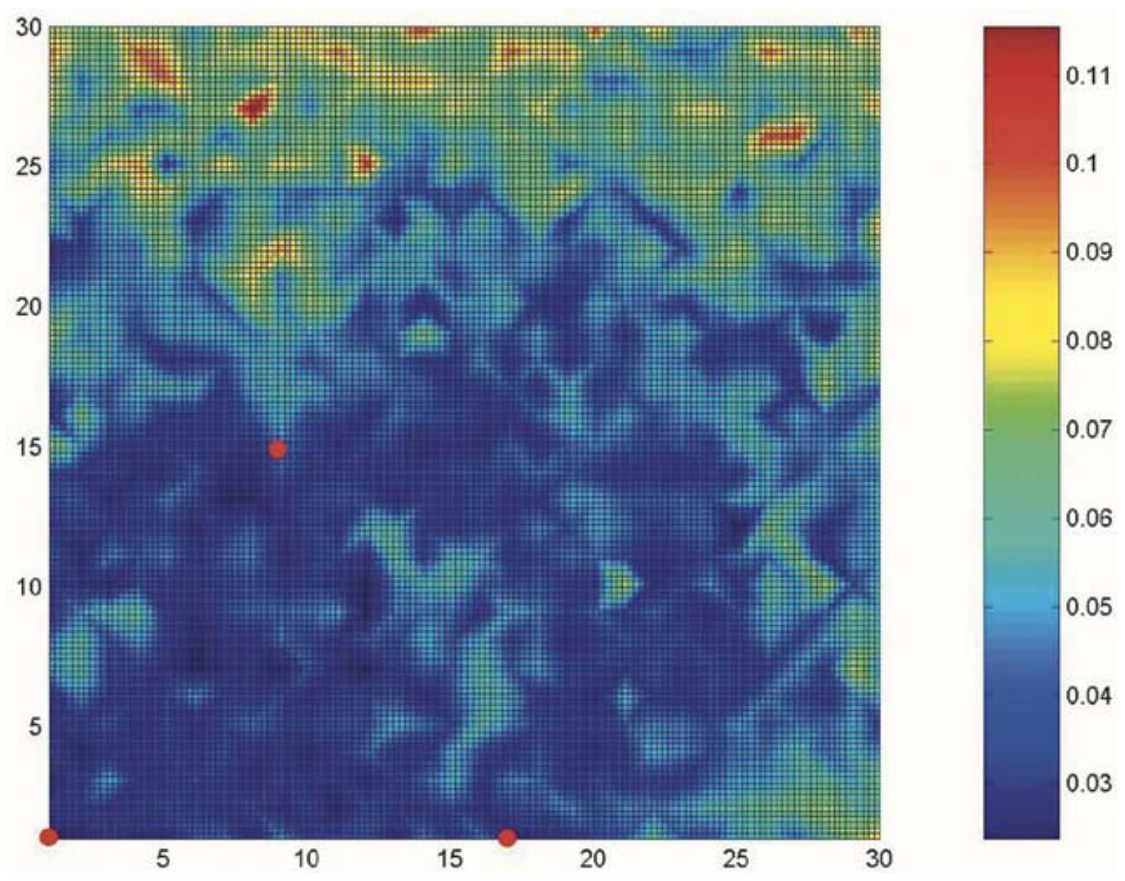

Fig. 14. Color-coded average nominal error over the admissible region as a function of $(x, y)$ position in the case of Gaussian distributed measurement error, winter conditions. The error chromatic scale is in $\mathrm{Km}$. Buoy locations are the red circles. Scales on the $x$ and $y$ axis are in $\mathrm{Km}$.

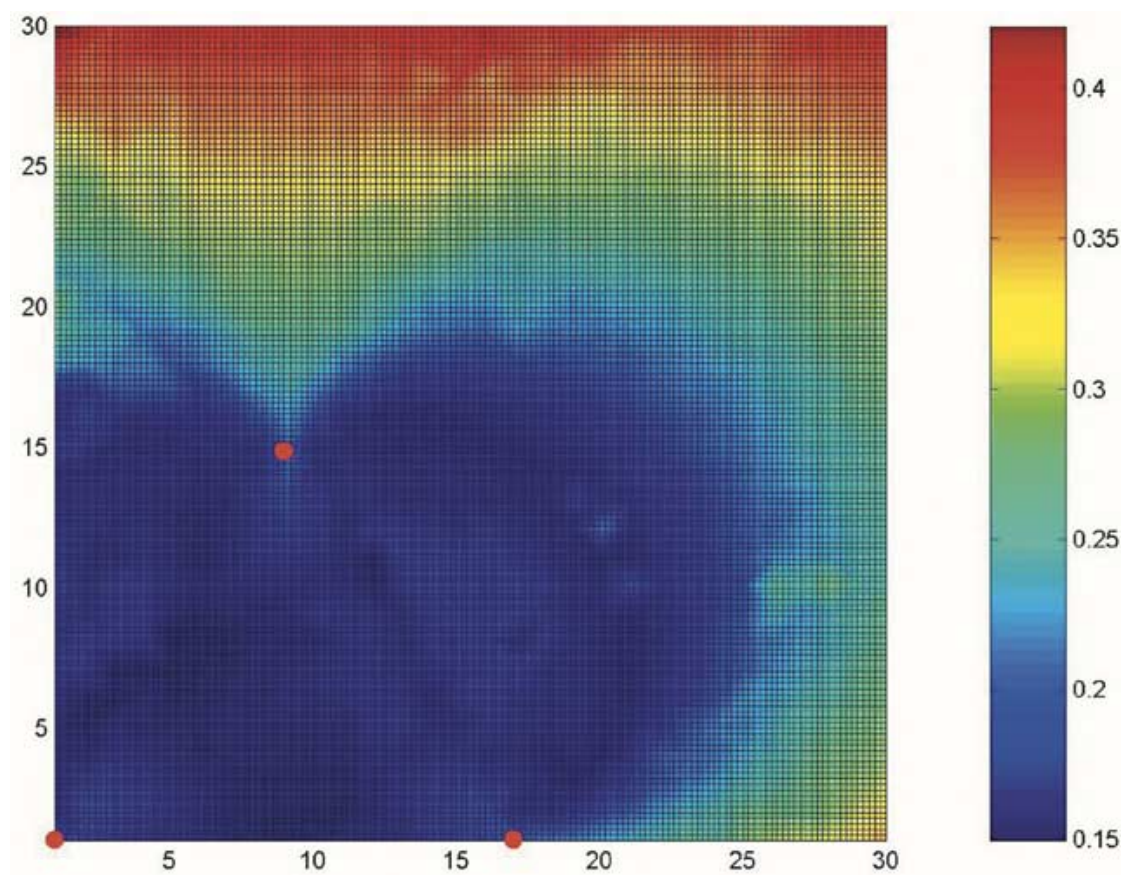

Fig. 15. Color-coded average worst-case error over the admissible region as a function of $(x, y)$ position in the case of Gaussian distributed measurement error, winter conditions. The error chromatic scale is in Km. Buoy locations are the red circles. Scales on the $x$ and $y$ axis are in $\mathrm{Km}$.

creases with range outside the buoys triangle area, and it is best along privileged directions, corresponding to the perpendicular axes to the triangle sides. For this configuration this result is also similar to that obtained by standard triangulation. The nominal error pattern distribution exhibits the same behavior, but it is more irregular, as already observed.

The Gaussian uncertainty case can be compared with the results reported by [21], where classical triangulation algorithms have been employed together with Gaussian distributed mea- surement errors (see Table I, where two specific vehicle locations are considered). Even if one accounts for the two-way travel time configuration in [21], it can be observed that the nominal errors provided by the set-membership approach are slightly smaller than those of the standard triangulation algorithm. Moreover, the set-membership procedure, by construction, does not have the pitfalls of triangulation algorithms, in which for a non negligible set of spatial regions, failure of the algorithm has been reported [21]. From this comparison it can 
TABLE I

COMPARISONS OF LOCALIZATION ALGORITHMS AT TWO SPECIFIC VEHICLE LOCATIONS (WINTER CONDITIONS)

\begin{tabular}{c|c|c|c|c|c|c}
\hline \multicolumn{2}{c|}{$\begin{array}{c}\text { Vehicle } \\
\text { position }(\mathrm{km})\end{array}$} & $\begin{array}{c}\text { (Mozzone } \text { et al., 2000a) } \\
\text { (2-way travel time) }\end{array}$ & \multicolumn{2}{c|}{$\begin{array}{c}\text { This work } \\
\text { (uniform noise) }\end{array}$} & \multicolumn{2}{c}{$\begin{array}{c}\text { This work } \\
\text { (Gaussian noise) }\end{array}$} \\
\hline$X$ & $Y$ & $\begin{array}{c}\text { nominal } \\
\text { error }(\mathrm{m})\end{array}$ & $\begin{array}{c}\text { nominal } \\
\text { error }(\mathrm{m})\end{array}$ & $\begin{array}{c}\text { maximum } \\
\text { error }(\mathrm{m})\end{array}$ & $\begin{array}{c}\text { nominal } \\
\text { error }(\mathrm{m})\end{array}$ & $\begin{array}{c}\text { maximum } \\
\text { error }(\mathrm{m})\end{array}$ \\
\hline 8 & 4.3 & 89 & 68.8 & 163.0 & 38.1 & 166.8 \\
\hline 23 & 10 & 115 & 65.4 & 164.0 & 35.9 & 181.6 \\
\hline
\end{tabular}

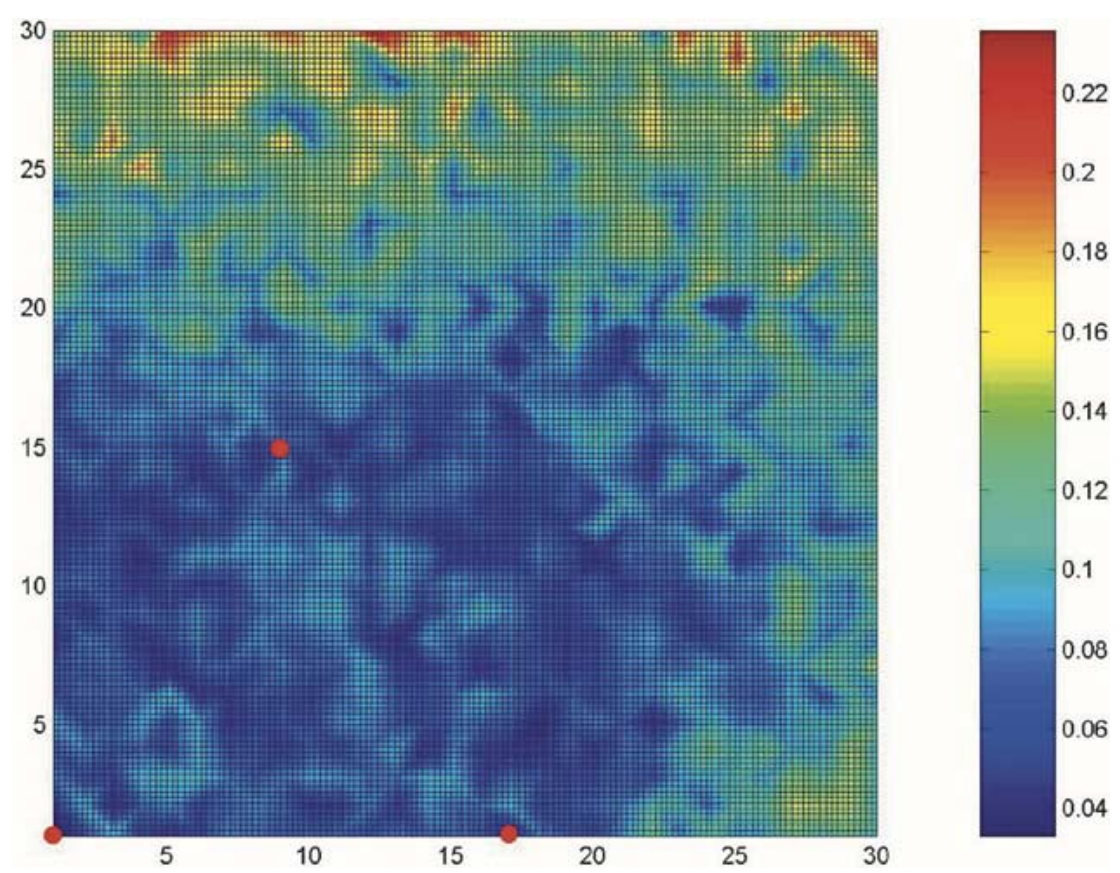

Fig. 16. Color-coded average nominal error over the admissible region as a function of $(x, y)$ position in the case of uniformly distributed measurement error, summer conditions. The error chromatic scale is in Km. Buoy locations are the red circles. Scales on the $x$ and $y$ axis are in $\mathrm{Km}$.

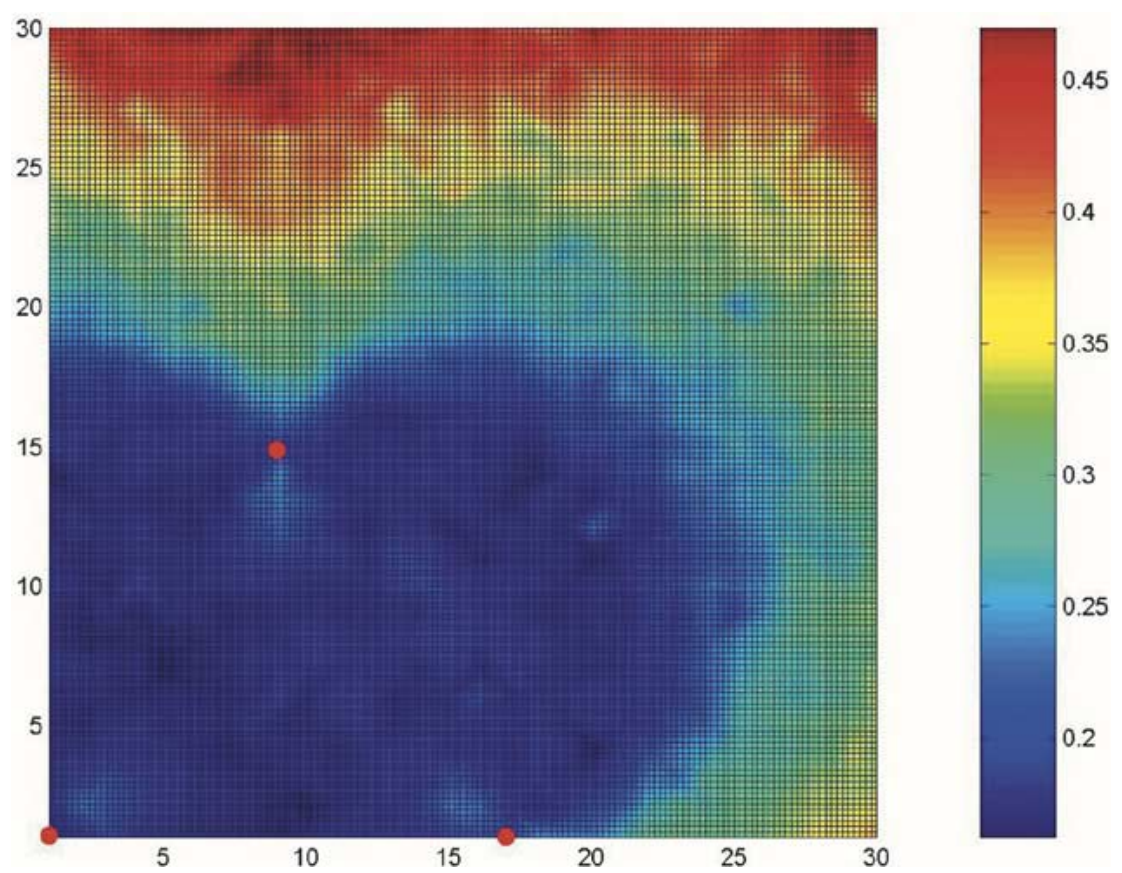

Fig. 17. Color-coded average worst-case error over the admissible region as a function of $(x, y)$ position in the case of uniformly distributed measurement error, summer conditions. The error chromatic scale is in $\mathrm{Km}$. Buoy locations are the red circles. Scales on the $x$ and $y$ axis are in $\mathrm{Km}$. 


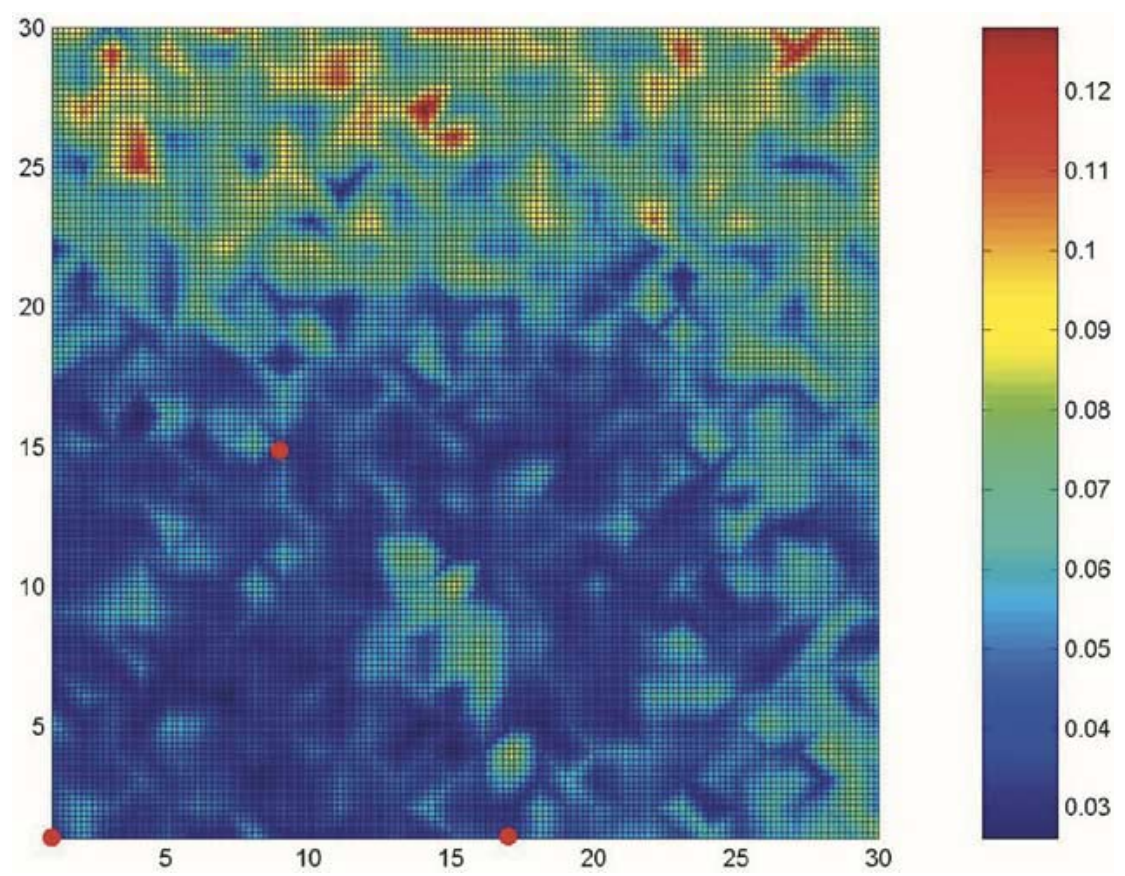

Fig. 18. Color-coded average nominal error over the admissible region as a function of $(x, y)$ position in the case of Gaussian distributed measurement error, summer conditions. The error chromatic scale is in Km. Buoy locations are the red circles. Scales on the $x$ and $y$ axis are in $\mathrm{Km}$.

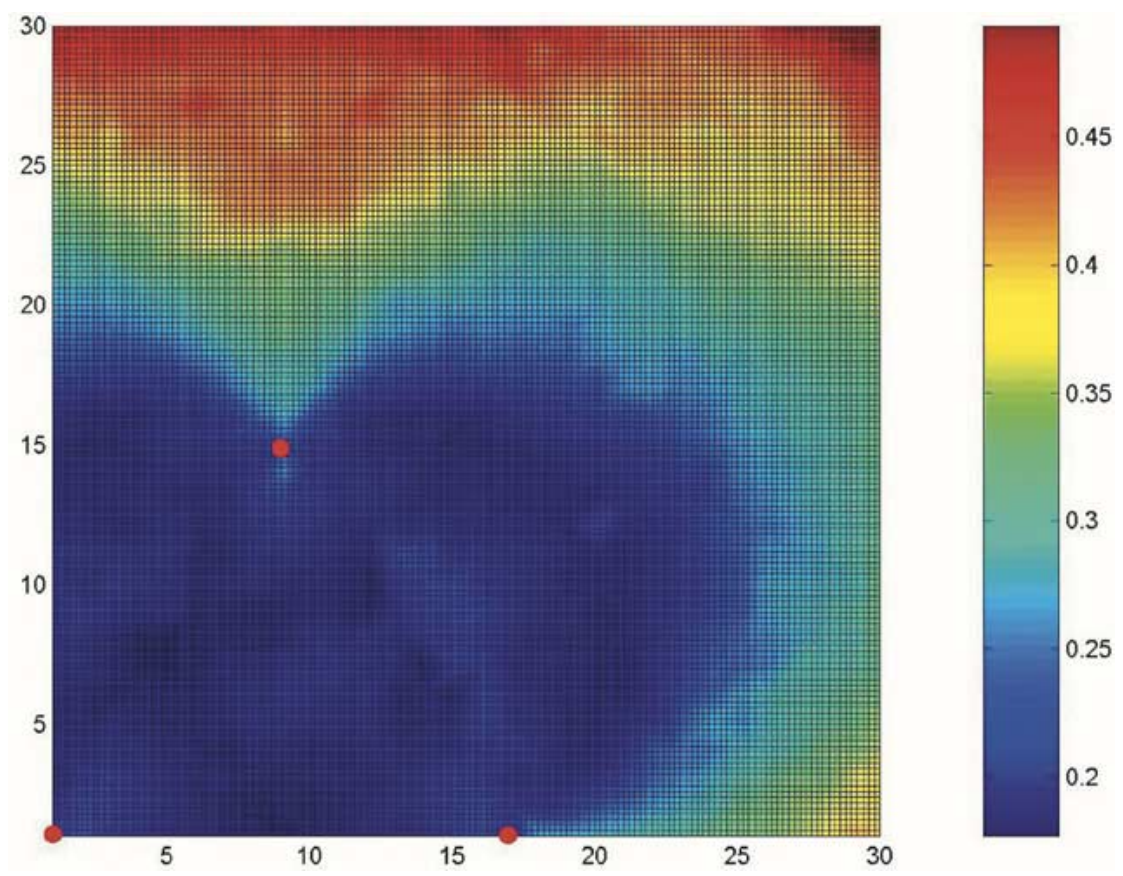

Fig. 19. Color-coded average worst-case error over the admissible region as a function of $(x, y)$ position in the case of Gaussian distributed measurement error, summer conditions. The error chromatic scale is in Km. Buoy locations are the red circles. Scales on the $x$ and $y$ axis are in Km.

be concluded that the set-membership approach offers a more robust localization procedure without degradation in the localization performance. The price to pay in this case is the computational burden of the set-membership procedure, which is definitely more time consuming of the standard approach.

A "summer condition" case is now considered, with a sound speed profile assumed as in Fig. 3. The measurement noise $e_{i}$ has been taken in a first case as uniformly distributed over the interval $[-66,66] \mathrm{ms}$, and i.i.d. for every buoy; in a second case the error has been taken as Gaussian i.i.d. for every buoy, with standard deviation $\sigma=22 \mathrm{~ms}$. In both cases, the localization algorithm is given information on the worst-case measurement error $E_{i}=66 \mathrm{~ms} \forall i$. Gridding and pseudoMonte Carlo analysis have been performed as in the winter conditions case. Results are reported in Figs. 16-19. They show that the nominal errors have not significantly changed with respect to the winter condition case, while the worst-case errors are larger. This is in agreement with expectation, since the procedure for computing 
TABLE II

COMPARISON OF LOCALIZATION AlgORITHMS AT Two SPECIFIC VEHIClE LOCATIONS (SUMMER CONDITIONS)

\begin{tabular}{c|c|c|c|c|r}
\hline \multicolumn{2}{c|}{$\begin{array}{c}\text { Vehicle } \\
\text { position }(\mathrm{km})\end{array}$} & \multicolumn{2}{c|}{$\begin{array}{c}\text { This work } \\
\text { (uniform noise) }\end{array}$} & \multicolumn{2}{c}{$\begin{array}{c}\text { This work } \\
\text { (Gaussian noise) }\end{array}$} \\
\hline$X$ & $Y$ & $\begin{array}{c}\text { nominal } \\
\text { error }(\mathrm{m})\end{array}$ & $\begin{array}{c}\text { maximum } \\
\text { error }(\mathrm{m})\end{array}$ & $\begin{array}{c}\text { nominal } \\
\text { error }(\mathrm{m})\end{array}$ & $\begin{array}{c}\text { maximum } \\
\text { error }(\mathrm{m})\end{array}$ \\
\hline 8 & 4.3 & 72.2 & 188.8 & 42.7 & 275.4 \\
\hline 23 & 10 & 74.9 & 208.9 & 48.1 & 289.0 \\
\hline
\end{tabular}

the bounding box in the variable sound speed profile case is generally more conservative than in the constant sound speed profile case. Moreover, the procedure will not affect the center of the box, but the volume of the box itself. Table II reports the results obtained in the summer condition case, at the same vehicle locations considered in Table I. The same differences between the uniform and the Gaussian case observed in the winter condition case are repeated in this case. The qualitative spatial distribution of the localization error is analogous to that of the winter condition case.

\section{CONCLUSION}

A localization algorithm for AUVs with measurements from a sparse field of acoustic buoys has been presented. The algorithm is based on set-membership estimation theory: no statistical assumptions on the disturbances are made, but only the worst-case bounds on the measurement errors must be available. Performance comparison with other methods relying on statistical hypotheses is not easily accomplished, due to the diversity of the approaches. However, when comparison is possible, the localization error distribution computed with the set-membership approach is similar to that obtained by standard techniques, and computationally more robust, at the price of a more demanding computational burden. As a bonus, the set-membership algorithm produces also the region in which the vehicle is guaranteed to be located.

It is believed that the algorithm proposed may represent a significative in-the-field alternative to the ones based on statistical error characterization for those situations in which the disturbances cannot be well characterized or anticipated, and it is imperative to exactly bound the region to which the vehicle belongs.

\section{REFERENCES}

[1] E. Bai, H. Cho, R. Tempo, and Y. Ye, "Optimization with few violated constraints for linear bounded error parameter estimation," IEEE Trans. Autom. Control, vol. 47, no. 7, pp. 1067-1077, 2002.

[2] C. Bechaz and H. Thomas, "Gib system: the underwater GPS solution," in Proc. 5th Europ. Conf. Underwater Acoustics, Lyon, France, 2000.

[3] J. Borenstein, B. Everett, and L. Feng, Navigating Mobile Robots: Systems and Techniques. Wellesley, U.K.: AK Peters, 1996.

[4] A. Caiti, "Seafloor exploration with parametric sonar systems: current issues and challenges for underwater vehicles," in Proc. IEEE Conf. Control Applications, Trieste, Italy, 1998.

[5] A. Caiti, A. Garulli, F. Livide, and D. Prattichizzo, "Set membership acoustic tracking of autonomous underwater vehicles," Acta Acustica United With Acustica, vol. 88, no. 5, pp. 648-652, 2002.

[6] - "Set-membership localization and tracking of autonomous underwater vehicles," in Proc. 6th Europ. Conf. Underwater Acoustics, Gdansk, Poland, 2002.
[7] _ - "Set-membership localization and tracking of autonomous underwater vehicles by a sparse field of floating acoustic buoys," in Proc. 14th IFAC World Conf., Barcelona, Spain, 2002.

[8] L. Collin, S. Azou, K. Yao, and G. Burel, "On spatial uncertainty in a surface long base-line positioning system," in Proc. 5th Europ. Conf. Underwater Acoustics, Lyon, France, 2000.

[9] A. Garulli and A. Vicino, "Set membership localization of mobile robots via angle measurements," IEEE Trans. Robot. Autom., vol. 17, no. 4, pp. 450-463, 2001.

[10] J. P. Hermand, P. Boni, E. Michelozzi, P. Guerrini, M. Agate, A. Borruso, A. D’Argenio, D. DiMaio, C. Loiacono, M. Mancuso, and M. Scannavino, "Geoacoustic inversion with drifting buoys: Enverse 1997-98 experiment," in Experimental Acoustic Inversion Methods for Exploration of the Shallow Water Environment, A. Caiti, J. P. Hermand, S. M. Jesus, and M. B. Porter, Eds. Dordrecht, The Netherlands: Kluwer Academic, 2000.

[11] F. Jensen, W. Kuperman, M. B. Porter, and H. Schmidt, Computational Ocean Acoustics. New York: Amer. Inst. Phys., 1994.

[12] J. S. Julier, J. K. Uhlmann, and H. F. Durrant-Whyte, "A new approach for filtering nonlinear systems," in Proc. Amer. Control Conf., Seattle, Washington, 1995, pp. 1628-1632.

[13] J. J. Leonard and H. F. Durrant-Whyte, "Mobile robot localization by tracking geometric beacons," IEEE Trans. Robot. Autom., vol. 7, no. 3, pp. 376-382, 1991.

[14] T. S. Levitt and D. T. Lawton, "Qualitative navigation for mobile robots," Artif. Intell., vol. 44, no. 3, pp. 305-360, 1990.

[15] D. Meizel, A. Preciado-Ruiz, and E. Halbwachs, "Estimation of the location of a mobile robot: geometric approaches," in Bounding Approaches to System Identification, M. Milanese, J. P. Norton, H. Piet-Lahanier, and E. Walter, Eds. London, U.K.: Plenum, 1996.

[16] M. Milanese and A. Vicino, "Optimal estimation theory for dynamic systems with set membership uncertainty: an overview," Automatica, vol. 27, no. 6, pp. 997-1009, 1991.

[17] M. Milanese, J. P. Norton, H. Piet-Lahanier, and E. Walter, Eds. Bounding Approaches to System Identification. New York: Plenum, 1996.

[18] E. M. Mouaddib and B. Marhic, "Geometrical matching for mobile robot localization," IEEE Trans. Robot. Autom., vol. 16, no. 5, pp. 542-552, 2000.

[19] L. Mozzone and S. Bongi, "Localization and fusion of echoes with deployable multistatic active sonar: evaluation of feasibility using experimental data," in Proc. 4th Europ. Conf. Underwater Acoustics, Rome, Italy, 1998.

[20] L. Mozzone, P. Lorenzelli, A. Caiti, and S. Bongi, "Acoustic localization of marine crafts with multistatic deployable sonars," in Proc. 5th IFAC Conf. Manoeuvring Control Marine Crafts, Aalborg, Denmark, 2000.

[21] —_ "Target motion analysis with multiple distributed acoustic sensors," in Proc. 5th Europ. Conf. Underwater Acoustics, Lyon, France, 2000.

[22] J. S. Stambaugh and R. B. Thibault, "Navigation requirements for autonomous underwater vehicles," Navigation, 1992.

[23] N. Storkensen, J. Kristensen, A. Indreeide, J. Seim, and T. Glancy, "Hugin-UUV for seabed survey," Sea Technol., 1998.

[24] K. T. Sutherland and W. B. Thompson, "Localizing in unstructured environments: dealing with the errors," IEEE Trans. Robot. Autom., vol. 10, no. 6, pp. 740-754, 1994.

[25] S. T. Tuohy, J. J. Leonard, J. G. Bellingham, N. M. Patrikalakis, and C. Chryssostomidis, "Map based navigation for autonomous underwater vehicles," Int. J. Offshore and Polar Eng., 1996.

[26] X. Yun, E. R. Bachmann, R. B. McGhee, R. H. Whalen, R. L. Roberts, R. G. Knapp, A. J. Healey, and M. J. Zyda, "Testing and evaluation of an integrated gps/ins system for small auv navigation," IEEE J. Ocean. Eng., vol. 24, no. 3, pp. 396-404, 1999. 


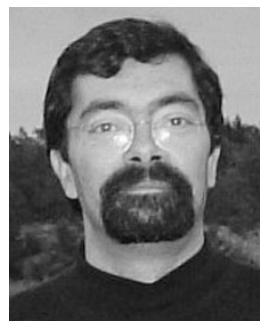

Andrea Caiti (M'92-SM'04) received the Laurea degree in electronic engineering from the University of Genova, Genova, Italy, in 1988.

From 1989 to 1994, he served as a Staff Scientist with the Saclant Undersea Research Center in the Seafloor Acoustics Group, La Spezia, Italy. In 1994, he joined the Italian University System, with assignments from the Universities of Genova, Pisa, and Siena, Italy. He is currently Associate Professor with the University of Pisa, with teaching assignments in system and control theory, system identification, and industrial automation. Since 2001, he has also been Director of the Italian Interuniversity Center of Integrated Systems for the Marine Environment (ISME), Genova, Italy. His research interests are focused on inverse problems, data processing, model estimation, and system identification, with applications in the field of robotics and underwater systems.

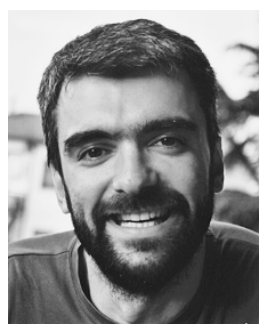

Andrea Garulli (M'95-SM'03) was born in Bologna, Italy, in 1968. He received the Laurea degree in electronic engineering from the Università di Firenze. Firenze, Italy, in 1993 and the Ph.D. degree in system engineering from the Università di Bologna, Bologna, Italy, in 1997.

In 1996, he joined the Dipartimento di Ingegneria dell'Informazione, Università di Siena, Siena, Italy, where he is currently an Associate Professor. His present research interests include robust identification and estimation, robust control, LMI-based optimization, mobile robotics, and autonomous navigation. He is an author of more than 70 technical publications and coeditor of Robustness in Identification and Control (New York: Springer, 1999).

Dr. Garulli has been a member of the Conference Editorial Board of the IEEE Control Systems Society and presently serves as Associate Editor for the IEEE TRANSACTIONS ON AUTOMATIC CONTROL.
Flavio Livide, photograph and biography not available at the time of publication.

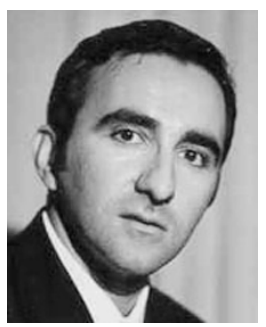

Domenico Prattichizzo (M'93) received the M.S. degree in electronics engineering and the Ph.D. degree in robotics and automation from the University of Pisa, Pisa, Italy, in 1991 and 1995, respectively.

Since 2002, he has been an Associate Professor of robotics and automation with the University of Siena, Siena, Italy. In 1994, he was a Visiting Scientist with the Artificial Intelligence Laboratory, Massachusetts Institute of Technology, Cambridge. Since 1992, he has been a Research Associate with the Robotics and Automation Group of the Centro "E.Piaggio" of the University of Pisa. He is coeditor of Control Problems in Robotics, vol. 4, STAR, Springer Tracks in Advanced Robotics (New York: Springer Verlag, 2003). He is coauthor of more than 100 papers in the area of robotics and automation.

Dr. Prattichizzo was coorganizer and chairman of many sessions on robotics and control theory in many International Conferences. He was co-chair of the Second IEEE International Workshop on Control Problems in Robotics and Automation, Las Vegas, NV, in December 2002. He serves as a Member of the Editorial Board of the IEEE TRANSACTIONS ON ROBOTICS, the IEEE TRANSACTIONS ON CONTROL AND SYSTEMS TECHNOLOGIES and of the Journal of Dynamics of Continuous, Discrete and Impulsive Systems (DCDIS) Series B: Application and Algorithms, and as a Member of the Conference Editorial Board of the IEEE Control System Society. 